BULLETIN Bulletin hispanique

HISPANIQUE Université Michel de Montaigne Bordeaux

118-1 | 2016

La Guerre Civile espagnole aujourd'hui (1936-2016)

\title{
Invariación y adecuación en teoría de la
} traducción

metalexicografía en el Dictionnaire de la conversation et de la lecture

(1835) y en la Enciclopedia moderna (1852)

Ignacio Ahumada

\section{OpenEdition}

Journals

Edición electrónica

URL: http://journals.openedition.org/bulletinhispanique/4388

DOI: $10.4000 /$ bulletinhispanique.4388

ISSN: 1775-3821

Editor

Presses universitaires de Bordeaux

Edición impresa

Fecha de publicación: 15 julio 2016

Paginación: 305-318

ISBN: $979-10-300-0058-0$

ISSN: 0007-4640

Referencia electrónica

Ignacio Ahumada, «Invariación y adecuación en teoría de la traducción », Bulletin hispanique [En línea], 118-1 | 2016, Publicado el 15 julio 2019, consultado el 08 septiembre 2019. URL : http://

journals.openedition.org/bulletinhispanique/4388; DOI : 10.4000/bulletinhispanique.4388 


\title{
Invariación y adecuación en teoría de la traducción: metalexicografía en el Dictionnaire de la conversation et de la lecture (1835) y en la Enciclopedia moderna $(1852)^{*}$
}

\author{
IgNaCio Ahumada \\ Consejo Superior de Investigaciones Científicas - Madrid (España)
}

La traduction-adaptation de l'article encyclopédique dictionnaire - qu'avait publié en 1835, dans la Dictionnaire de la conversation et de la lecture, l'érudit Jean Baptiste Pellissier - est considérée, à la suite de sa publication dans la Enciclopedia moderna (1852), comme le premier témoignage, général et important pour son histoire, de la métalexicographie de langue espagnole.

Mots-clés: théorie linguistique, linguistique romane, lexicographie encyclopédique hispano-française, métalexicographie de la langue espagnole.

La traducción-adaptación del artículo enciclopédico dictionnaire publicado en el Dictionnaire de la conversation et de la lecture (1835), y cuya autoría se debe al estudioso francés Jean Baptiste Pellissier, representa, tras su publicación en la Enciclopedia moderna (1852), el primer testimonio general y con cierta entidad en la historia de la metalexicografía del español.

Palabras claves: lingüística teórica, lingüística románica, lexicografía enciclopédica hispano-francesa, metalexicografía de la lengua española.

The translation-adaptation of the encyclopaedic entry «dictionnaire» published in the "Dictionnaire de la conversation et de la lecture» (1835), the author of which is the French scholar Jean Baptiste Pellissier, is presently recognized after its publication in the «Enciclopedia moderna» (1852), as the first general and important testimony of the Spanish meta-lexicology in the history.

Keywords: Theoretical Linguistics, Romance Linguistics, Spanish-French Encyclopedic Lexicography, Spanish Language Metalexicography.

* Este trabajo se encuadra dentro de los llevados a cabo para el proyecto Diccionario bibliográfico de la metalexicografía del español, 2006-2010, proyecto que goza de una ayuda del Ministerio de Ciencia e Innovación. Plan Nacional de I+D+i (2008-2011). Convocatoria 2010. Proyecto núm. FF2010-19702.

Bulletin Hispanique, Tome 118, n 1 - juin 2016 - p. 305-318. 


\section{INTRODUCCIÓN}

El método traductor, siempre de límites difusos y con variantes para todos los gustos ${ }^{1}$, no deja de despertar el más vivo interés cuando nos acercamos a textos de nuestro pasado cultural, sobre todo si nos hacemos cargo de la asepsia con la que el traductor debe enfrentarse a los textos científico-técnicos, bien sean estos en lenguas culturalmente alejadas bien lo sean en lenguas de cultura compartida. En el caso que nos ocupa se trata de un texto francés del siglo XIX y su traslado al público español en un momento especialmente complejo dentro de una disciplina en la que apenas si existían unos precedentes teóricos de especial relevancia. La metalexicografía o teoría sobre los diccionarios no pasaba en nuestra bibliografía nacional por otra cosa que no fueran referencias aisladas en los prólogos de algunos diccionarios o en escritos filológicos al uso (cf. Ahumada, 2008).

Si la lexicografía francesa de iniciativa privada, frente a la lexicografía oficial (Académie Française), ha representado en el país vecino la lexicografía por excelencia, en Espańa nos encontramos con un panorama diferente: la Real Academia Española vio amenazada la centenaria hegemonía de su diccionario general ante proyectos lexicográficos como el Panléxico (1842-1845) de Juan Peñalver, la exhaustiva ampliación de Vicente Salvá (1846 y 1847) o el ímpetu arrollador del Diccionario nacional de Ramón Joaquín Domínguez (1846$1847)^{2}$.

Ante una situación como esta tiene lugar en España la publicación de la primera enciclopedia completa -según mis datos- después del Antiguo Régimen ${ }^{3}$. Me refiero a la traducción y adecuación al español de la nueva edición de la Encyclopédie moderne (1846-1863), bajo la dirección de Léon Renier, y conocida en Espańa como la Enciclopedia moderna o de Mellado (1851-1855).

1. Aunque el binomio traducción literal (verbum pro verbo)/traducción libre (sensum ad sensu) nos ayude sobremanera a simplificar las cosas, especialmente en el aula.

2. Debe tenerse en cuenta que tanto el proyecto de Juan Peñalver (Pan-lexique (1829) de Charles Nodier) como el de Ramón Joaquín Domínguez (Dictionnaire national (1843) de Louis Nicolas Bescherelle) son de clara inspiración francesa (cf. Ahumada, 2011: 61-63 y Seco, 2003: 294-295). Tanto Manuel Núñez de Taboada como Vicente Salvá eran conocedores de la mediata e inmediata lexicografía francesa, ambos estaban radicados en París cuando publicaron su revisión del diccionario académico: Diccionario de la lengua castellana (1825) y Nuevo diccionario (1846/1847). Valga como ejemplo temprano para el caso de la lexicografía francesa la revisión de Le Dictionnaire universel (1690) de Antoine Furetière por parte de Basnage de Baural (1701) y los jesuitas de Trévoux (1704), cuyo reflejo en la lexicografía espańola no es otro que la única muestra no académica de nuestro siglo XVIII: el Diccionario castellano con las voces de ciencias y artes (1786-93) del padre jesuita Esteban de Terreros. Y para el siglo XIX baste citar la revisión del Dictionnaire universel de la langue française (1800) de P. C. V. Boiste por el ya citado Ch. Nodier.

3. De la Encyclopedia metódica por orden de materias (1788-1794), del editor Antonio de Sancha, a partir de la Encyclopédie méthodique (1788-1832), del editor francés Panckoucke, tan solo se publicaron diez volúmenes del total de 167 que conformaban la edición original francesa. Como es sabido, el proyecto espańol se fue al traste tras la polémica suscitada por determinadas apreciaciones incluidas en el artículo «Espagne», cuya autoría se debe a Masson de Morvilliers, dentro del volumen Géographie (1788). 
Me propongo revisar el proceso de la traducción entre dos lenguas de naturaleza afín y culturalmente próximas a partir del análisis contrastivo del artículo diccionario en la Enciclopedia moderna de Mellado y en el Dictionnaire de la conversation et de la lecture (1835), referencia que fue de los traductores españoles toda vez que la Encyclopédie moderne carecía, en este caso, de una monografía dedicada a los repertorios lexicográficos.

La traducción y adecuación del artículo monográfico dictionnaire, firmado por Jean Baptiste Pellissier, representa con diferencia el primer testimonio cerrado y de envergadura de nuestra historia metalexicográfica.

\section{TeXtos METALEXICOGRÁficos}

El interés de los estudiosos por el objeto diccionario corre de forma paralela a la aparición de este tipo de obras en la Edad Moderna. En el tiempo, al lado de las reseñas de obras lexicográficas en gramáticas, retóricas o bien en repertorios bibliográficos (cf. Ahumada, 2007), figuran los prólogos, por un lado $;$; los artículos sobre voces metalexicográficas (diccionario, enciclopedia, vocabulario, lexicón, glosario, etc.) en obras enciclopédicas, por otro 5 . Nuestro interés se dirige hoy de manera exclusiva -como acabo de señalar- al artículo diccionario en el primer trabajo, según mis noticias, que se ocupa de dar un tratamiento enteramente metalexicográfico a la voz en cuestión.

Franz Josef Hausmann cita a Antoine Furetière y su Dictionnaire universel (1690) como uno de los primeros repertorios que incluye noticias de carácter enciclopédico en el artículo diccionario, si bien estas noticias no pasan de la mención de algunos diccionarios. Desde aquí traslada su revisión histórica hasta la obra enciclopédica por excelencia: Encyclopédie, ou Dictionnaire raisonné des sciences, des arts et des métiers (1751-1772) de J. Le Rond D'Alembert y Denis Diderot (Hausmann, 1989: 217a), en donde voces como diccionario, enciclopedia o definición constituyen verdaderos capítulos de un manual al uso.

\section{El artículo “dictionnaire” de Jean Baptiste Pellissier (1835)}

Del artículo o monografía que nos ocupa, disponemos de dos versiones de J. B. Pellissier publicadas con tan solo un año de diferencia. La primera de ellas

4. De los tres diccionarios españoles de iniciativa privada mencionados arriba tan solo el Nuevo diccionario (1846) de V. Salvá contiene, en mi opinión, un prólogo metalexicográfico. La razón es bien sencilla: el autor ha de justificar los criterios y el método empleado para aumentar el DRAE («Introducción del adicionador», pp. VII-XXXVII); en tanto que J. Peñalver y R. J. Domínguez adaptan el modelo francés sin más consideraciones de carácter teórico. De los entresijos del Panléxico conocemos algo más gracias a la polémica suscitada en la prensa madrileña de 1842 cuando se publicó el prospecto que adelantaba la publicación de la obra (cf. Ahumada, 2011: 59 y ss.).

5. Véase mi trabajo «Translation and ideology: neighborhood policy in Enciclopedia moderna (1851-1855). A propos the article diccionario», en M. ${ }^{a}$ M. Rivas Carmona y M. ${ }^{a}$ C. Balbuena Torezano (ed.), Cultural aspects of translation, Tübingen, Narr Verlag, 2013, pp. 1-12. 
responde a su colaboración en el Dictionnaire de la conversation et de la lecture, publicada en $1835^{6}$, en tanto que la segunda -con notables variantes- vio la luz en el Bulletin du Bibliophile de 1836 (Pellissier, 1835; Pellissier, 1836 y Pellissier, 1836a $)^{7}$.

El artículo monográfico dictionnaire, con una estructura muy poco o nada usual en la lexicografía de su tiempo, se sustenta en los dos grandes pilares sobre los que se levanta la literatura lexicográfica: de una parte, la historia de los diccionarios; de otra, la teoría sobre los diccionarios o metalexicografía ${ }^{8}$. Los planteamientos y consideraciones de una y otra partes se nos muestran como claros exponentes de su experiencia como lexicógrafo, dado que fue uno de los colaboradores en los trabajos preparatorios de la sexta edición del Dictionnaire de l'Académie Française (1835) (Quérard, 1835: s. v. $)^{9}$. La novedad de su aportación, según manifiesta el propio autor, radica antes en la presentación histórica de los diccionarios de las lenguas de Europa que en los aspectos estrictamente metalexicográficos:

Et qu'aucun livre jusqu'ici, à ma connaissance, n’a présenté l'historique des vocabulaires. Je vais donc essayer de remplir, sous ce rapport, une partie de la lacune que nous a laissée la science bibliographique (Pellissier, 1835: 469a).

Je n'ignore pas, d'ailleurs, combien le résultat de mes recherches laisse encore à désirer, mais je sais aussi quion trouve à peine quelques notions éparses; et fort incomplètes, sur les vocabulaires antérieurs au xviiie siècle, et j'ai lieu d'espérer que mon travail, pour imparfait qu'il puisse être, remplira, du moins, une partie de la lacune que existe à ce sujet dans la bibliographie (Pellissier, 1836: 122).

Esta primera parte («Notions historiques») se articula sobre dos centros neurálgicos: (a) la lexicografía griega y latina (clásica y medieval) con las aportaciones a la misma a partir del siglo XVI (los Estienne, por ejemplo) y (b) los distintos diccionarios franceses monolingües y bilingües. Estos dos puntos de referencia le permiten a J. B. Pellissier extender sus comentarios a las aportaciones realizadas por los países del entorno: Alemania, España, Inglaterra, Italia, Portugal, Suecia y Rusia, entre otros. Las descripciones más extensas corresponden, como es evidente, a la labor de los lexicógrafos franceses y de la Académie Française. Esto no obsta para que buena parte de los repertorios

6. El Dictionnaire de la conversation et de la lecture consta de 68 tomos en 34 vol. publicados en París entre 1832 y 1851. J. B. Pellissier no solo se ocupó de redactar el artículo «diccionario», sino que además se hizo cargo de determinados artículos de literatura medieval; no obstante, había sido colaborador de François Just Marie Raynouard en su Choix des poésies originales des troubadours (1816-1821).

7. Revista mensual publicada por el editor parisino Jacques Techener y en la que el también lexicógrafo Ch. Nodier jugaba un importante papel como colaborador.

8. Así por ejemplo en la Encyclopédie de J. L. D’Alembert y D. Diderot: el artículo dictionnaire es exclusivamente teórico, al igual que el artículo encyclopédie, por citar las dos monografías que se dedican a los dos campos de acción de la lexicografía como disciplina lingüística (lexicografía lingüística y lexicografía enciclopédica).

9. Esto no impedirá -como veremos- que muestre con severidad sus diferencias con los planteamientos metalexicográficos de la institución. 
europeos merezca un breve comentario, en unos casos; la sola mención, en otros $^{10}$.

En la segunda parte («Principales conditions d'un bon dictionnaire»), J. B. Pellissier resume en poco más de ocho páginas los puntos esenciales para la redacción de un diccionario monolingüe general. Los distintos apartados que somete a descripción y análisis, no exentos de crítica, se corresponden con los principales temas que preocupaban a la lexicografía de su tiempo. Aparte del consabido problema de la definición y la clasificación de acepciones (significado recto y variantes), uno de los asuntos que trata con mayor profusión se refiere a la presencia de autoridades en los diccionarios generales del entorno europeo. De ahí que destaque, y elogie sin parangón, el diccionario inglés de Samuel Johnson; o bien que reconozca los méritos del trabajo de la Accademia della Crusca, aun seńalando sus limitaciones en cuanto al número de autoridades (le tre corone); o por otro lado que mencione la dependencia del Diccionario de autoridades de la Academia Espańola con respecto al modelo italiano; no deja, sin embargo, de sorprender, la exquisita deferencia hacia la más reciente de las producciones europeas, el diccionario portugués de la Academia de Ciencias. En el punto de mira estaba - no lo oculta-, el Dictionnaire de l'Académie Française. En su opinión, el repertorio francés debería avalar las definiciones con citas, entre otro tipo de textos, de las obras más representativas de la literatura francesa.

La idea de nuestro autor sobre el trabajo lexicográfico, en fin, queda bien patente, cuando liberado del manual de estilo del Dictionnaire de la conversation, reelabora para el Bulletin du Bibliophile el artículo dictionnaire:

L'art de recueillir et de ranger dans un ordre systématique les mots d'une langue, en les expliquant par des définitions, dont le développement indique leurs acceptions diverses, éclaircies et précisées par des exemples, cet art, ou pour mieux dire, cette science toute d'érudition et de patience... (Pellissier, 1836: 119).

\section{El artículo “diccionario" en la Enciclopedia moderna (1852)}

La traducción-adaptación española, mutatis mutandis, se rige en esencia por el mismo principio racionalista que determina el planteamiento de J. B. Pellissier, tanto por lo que hace a la aceptación de los procesos históricos en la lexicografía europea como por los principios teóricos que deben presidir la redacción de un diccionario general monolingüe; porque hemos de tener en cuenta que si bien en el original francés la revisión histórica se articula sobre la oposición lexicografía monolingüe/lexicografía bilingüe -punto de partida inexcusable para la lexicografía europea-, tan solo el diccionario monolingüe general es principio y fin del análisis teórico planteado en la segunda parte del artículo.

10. Algo bien distinto a lo que encontramos en la Table alphabétique des dictionnaires (1758) y, por lo que respecta a los aspectos metalexicográficos, en «Question sur les dictionnaires» de Charles Bellet. 
Atendiendo al principio de invariación que debe presidir todo proceso traductor, la estructura del artículo original se respeta escrupulosamente. Apenas se pueden contar con los dedos de la mano las omisiones del traductor, las cuales atienden antes a ejemplos muy concretos relacionados con la lengua francesa que a cuestiones de índole general, como pudieran ser la recepción del diccionario inglés de Samuel Johnson en Francia o la ejemplificación con voces francesas alejadas de la realidad lingüística del español. Cosa bien distinta serán las amplificaciones sobre el devenir de la lexicografía española, la exaltación de la riqueza verbal del español o la denuncia sobre el abuso de los galicismos en el español escrito.

\section{INVARIACIÓN Y ADECUACIÓN CULTURAL}

El texto sujeto a análisis, dentro de la oposición lengua general/lengua especializada, se adscribe sin ninguna dificultad al género científico-técnico en su variante humanística. Internamente el texto sigue dos direcciones claramente diferenciadas: de una parte, nos encontramos frente a un discurso marcadamente histórico, determinado por una cronología concreta (la historia de los diccionarios); de otra, frente a un discurso teórico-práctico (teoría de la práctica lexicográfica).

\section{Omisiones: necesidades del Dictionnaire y el papel de la Académie Française}

Por lo que hace al apartado historiográfico, apenas dos párrafos fueron omitidos por el traductor español. En ambos casos las omisiones vienen motivadas - a mi juicio- por la especificidad de las mismas, dado que se trata de referencias muy concretas a la labor de la Académie Française. De un lado, la notable diferencia cualitativa del diccionario inglés de $S$. Johnson frente al académico francés; y de otro, una serie de recomendaciones generales sobre los criterios metalexicográficos que aplica la institución en su diccionario:

Sans hasarder aucune induction à cet égard, ce qui m'appartiendrait moins qu’à personne, je crois toutefois pouvoir regretter d'avance que cette docte corporation ait persisté à exclure les citations de nos meilleurs auteurs, et que, se bornant encore à constater l'état de la langue usuelle, elle n'ait pas adopté un plan moins restreint, un point de vue plus élevé, une exécution moins dénuée d'esprit philosophique. Alors, elle eût fait entrer dans son dictionnaire un plus grand nombre de termes d'arts et de sciences; elle n'aurait pas négligé totalement les étymologies; elle eût indiqué la racine des mots, les significations relatives des synonymes, la quantité prosodique des syllabes; elle aurait pu donner les solutions de toutes les difficultés grammaticales, et surtout rajeunir beaucoup d'expressions tombées à tort en désuétude, et qui, n'ayant point d'analogues, semblent, par leur utilité, mériter de reprendre leurs droits de bourgeoisie (Pellissier, 1835: 476a). 
Por esta misma línea discurren las omisiones bajo el epígrafe «Principales conditions d'un bon dictionnaire». Es en la conclusión del artículo donde J. B. Pellissier dedica unas palabras al papel de la Académie Française ante las innovaciones a las que se sujeta el uso de francés en mano de sus «hardis novateurs»:

Le premier devoir de l'Académie est de ramener la langue dans les limites raisonnables que ces modèles on su toujours respecter sans rien perdre de leur essor et de leurs prodigieux avantages. Le principe constitutif de l'Académie française doit être en effet un principe conservateur (Pellissier, 1835: 484b).

\section{Amplificaciones: diccionarios españoles y defensa del español}

Las referencias de J. B. Pellissier a los diccionarios del español no van más allá de la simple mención junto a los repertorios de las lenguas modernas de su entorno, pues -como señalo arriba- el eje de sus comentarios historiográficos gira alrededor de la lexicografía greco-latina y de la lexicografía francesa. Contamos, pues, con alusiones a la obra bilingüe de Antonio de Nebrija, al Tesoro de la lengua castellana o española de Sebastián de Covarrubias y al diccionario académico: «l'Académie de Madrid donna en 1726 et années suivantes, un ouvrage fait à l'instar de celui de la Crusca, avec des exemples tirés des meilleurs auteurs espagnols» (Pellissier, 1835: 475b).

Las amplificaciones en consecuencia discurren, a grandes rasgos, por la lexicografía latino-española (Manuel de Valbuena), la lexicografía etimológica (S. de Covarrubias, Francisco del Rosal y Ramón Cabrera), la lexicografía monolingüe general no académica (Esteban de Terreros, Juan Peñalver, Prudencio J. de Vega, Ramón Joaquín Domínguez y José Caballero) o bien la lexicografía bilingüe bidireccional entre el francés y el español (Antonio Rotondo y autor anónimo).

De haber tenido J. B. Pellissier noticias, como las tenía de A. de Nebrija, dudo que no hubiera dedicado una mención al Diccionario universal latinoespañol (1793) de Manuel de Valbuena, puesto que no podríamos encontrar una obra más contraria al espíritu que anima el trabajo del lexicógrafo francés, para quien las citas literarias se hacen imprescindibles en cualquier diccionario que se precie:

Viene a ser mi diccionario el de Forcellini, sin las citas que le hacen tan voluminoso, reducidos los lugares de los autores a las meras frases y abreviadas lo más que ha sido posible sus definiciones e interpretaciones; pero de voces no falta ninguna, como no haya sido por descuido (Valbuena, 1793: col. 1004) ${ }^{11}$.

Baste con recordar a raíz de esta declaración cómo la supresión de las autoridades tras el primer diccionario académico (1726-1739) empobrece

11. Este diccionario fue el de mayor éxito de entre los de su tiempo: Valbuena, Manuel de (1793): Diccionario universal latino-españollespañol-latino, Madrid, Cano. 
nuestra particular trayectoria lexicográfica, ya se refiera a nuestros diccionarios monolingües ya a nuestros diccionarios bilingües ${ }^{12}$. Esto no quita que sea en estos años y en los inmediatos cuando contemos con lexicógrafos como Adolfo de Castro y Aniceto de Pagés que organicen sus diccionarios a partir de $\operatorname{citas}^{13}$.

El interés del anónimo traductor por los diccionarios etimológicos del español no deja de sorprender a propios y extraños. El estudioso francés, guiado con toda probabilidad por el título y la proximidad temporal, sitúa el Thrésor de la langue françoyse (1606) de Jean Nicot al lado del Tesoro de la lengua castellana o española (1611) de S. de Covarrubias, cuando nos encontramos ante dos obras de diferente factura lexicográfica. En tanto la primera está considerada por la crítica como un diccionario semibilingüe del francés, la obra del canónigo conquense, cuyo hilo conductor es la etimología, responde a una suerte de repertorio monolingüe que incluye tanto voces espańolas como una nutrida nomenclatura sobre geografía e historia sacra y profana (Ahumada, 2011a) ${ }^{14}$. La amplificación por parte del traductor se refiere a segunda edición del Tesoro (1674) con las adiciones del padre Benito Remigio Noydens.

La segunda referencia de nuestra lexicografía etimológica se refiere a la obra, entonces inédita, de Francisco del Rosal, Origen y etimología de todos los vocablos originales de la lengua castellana (1601-1611) ${ }^{15}$. El comentario del anónimo traductor nos revela que el lexicógrafo malagueño Antonio Martínez del Romero había estudiado y extractado la obra para los trabajos preparatorios «de un diccionario general y etimológico de la lengua española que en unión de don Rafael María Baralt están escribiendo» (Anónimo, 1852: s. v. col. 1005) ${ }^{16}$.

12. Al decir de Voltaire, «un dictionnaire sans citations est un squelette».

13. Gran diccionario de la lengua española (1852-1855) y Gran diccionario de la lengua castellana (con ejemplos) de buenos escritores antiguos y modernos (1902-c. 1932), respectivamente. Sobre la lexicografía general del español con citas, véase la tesis doctoral, defendida recientemente en la Universidad Complutense de Madrid, de Eduardo Jacinto García, El principio de autoridad en los diccionarios generales del español (siglos XVIII-XX) (<http://eprints.ucm.es/20660/1/T34377. pdf $>$ ). Importa reconocer en este sentido un considerable número de obras menores (regionales y especializadas) en las que no falta el apoyo de sus definiciones en citas.

14. Me refiero a la obra conjunta del Tesoro, esto es, el volumen publicado en Madrid el año de 1611 con el Suplemento del propio S. de Covarrubias, y cuya edición conjunta e ilustrada la publicaron Ignacio Arellano y Rafael Zafra en 2006 bajo el sello editorial de la Universidad de Navarra-Iberoamericana-Vervuert-Real Academia Española-Centro para la Edición de Clásicos Españoles.

15. Manuscrito 6229 de la Biblioteca Nacional de España. El manuscrito del siglo XVIII cuenta con una edición facsimilar y un estudio introductorio a cargo de Enrique Gómez Aguado, Madrid, Consejo Superior de Investigaciones Científicas, 1992.

16. Antonio Martínez del Romero como lexicógrafo fue autor de un incompleto Dizionario italiano-spagnuolo e spagnuolo-italiano (1844-1847) (Martínez Egido, 2010) y de un "Glosario compuesto de varias palabras cuya esplicación es necesaria para la inteligencia del catálogo que antecede [Catálogo descriptivo, artístico e histórico de los objetos de la Armería Real de Madrid]" (1849) y como traductor de la lengua francesa cuenta en su haber, entre otras traducciones, con Aventuras de Tom Pouce, Madrid, Hortelano, 1845; Mme Cottin, Matilde o Memorias sacadas de la historia de las cruzadas (1847), así como del divulgador de la ciencia Camille Flammarion, Les mondes imaginaires et les mondes réels y La pluralité des mondes habités, ambas publicadas en Madrid por Gaspar y Roig en 1873. 
Se trataba del malogrado proyecto Diccionario matriz de la lengua castellana, del que solo llegó a publicarse en 1850 una muestra (prospecto) del mismo. El polígrafo venezolano R. M. ${ }^{a}$ Baralt pretendía dirigir y coordinar un proyecto lexicográfico de vastos alcances sobre la lengua española, un diccionario etimológico, general, histórico y onomasiológico, en el que A. Martínez del Romero no hubiera figurado como único colaborador ${ }^{17}$.

Y en última instancia, la obra póstuma de Ramón Cabrera, Diccionario de etimologías de la lengua castellana (1837), antes colección de voces con procedencia latina que, a pesar del título, repertorio general de nuestra lengua ${ }^{18}$.

Hasta los años en los que se publica la Enciclopedia moderna la actividad lexicográfica del español, si la comparamos, por ejemplo, con la francesa, había sido relativamente baja y de escasa originalidad. Los años en los que nos encontramos fueron decisivos para llevar a cabo iniciativas impensables en los ańos precedentes, iniciativas que no partieran de revisar el $D R A E$ vigente para aumentarlo o epitomizarlo, cuando no fuera para trasladarlo con pocas o muy pocas diferencias respecto del original. En estos años cruciales se está gestando una nueva lexicografía general, de manifiesta inspiración francesa -claro está-, y paralelamente tuvo lugar la aparición de una prometedora lexicografía regional española e hispanoamericana (Aragón y Cuba), como habíamos asistido a la edición de los primeros repertorios especializados de cierta envergadura (términos geográficos, de náutica o de minería, entre otros).

La adecuación del articulo dictionnaire a la lexicografía del español discurre -como veremos- por la mención de aquellos trabajos que de una manera u otra han marcado su impronta en la historia de nuestros diccionarios generales.

La obra del jesuita E. de Terreros sobresale de entre todas ellas. Se trata del único repertorio que merece un comentario, aunque este fuera negativo e injustificado, por parte de nuestro anónimo autor. La mirada del traductor se dirige hacia la inclusión gratuita en sus columnas de entradas ajenas al español de la época. Señala, con la mención expresa de que se trata de voces portuguesas, la voz acuador, y por extensión abnous:

Desde luego es estraña la admisión de la voz acuador, que aunque la usan los portugueses, no está admitida en la lengua española; voz que no debía mencionarse por cuanto el pez que en ella se indica, tiene en español su correspondencia, que es volador (Anónimo, 1852: col. 1009).

17. El Diccionario matriz de la lengua castellana (prospecto) cuenta con una edición facsimilar publicada en Maracaibo en 1957. Para el filólogo español afincado en Caracas Pedro Grases el rechazo de Bartolomé José Gallardo a su participación en el proyecto fue determinante para su paralización (cf. Grases, 1943: 40). Debe tenerse en cuenta que entre la lista de colaboradores de la Enciclopedia moderna figura R. M. a Baralt como responsable del artículo "Filología» (Mellado, 1851: VIII).

18. En la Biblioteca de la Real Academia Española, de la que fue miembro y director, destituido de manera fulminante al regreso de Fernando VII (1814), se conserva manuscrito su Diccionario del lenguaje antiguo castellano (cf. Zamora, 1999: 179a). 
Bastaba con haber consultado el tercer volumen del Diccionario castellano para constatar que el padre Terreros daba entrada a volante y volador como variantes españolas para denominar esta subespecie acuática ${ }^{19}$. De todas formas, acuador acabaría teniendo fortuna lexicográfica, aunque se tratara de una voz portuguesa, pues del padre Terreros la toman R. J. Domínguez (1846), los editores Gaspar y Roig (1853), Elías Zerolo (1895) y Manuel Rodríguez Navas $(1918)^{20}$.

El Diccionario castellano con las voces de ciencias y artes (1786-1788) es de una complejidad sin límites a pesar del extenso prólogo que lo precede. Pero de la misma manera que el traductor ha tomado como referencia acuador podría haberlo hecho con otras muchas entradas: sabah, romescot, Viztzilipuztli, Huchilobos o rum. Hace casi dos décadas que Félix San Vicente habló de la solución 'omnicomprensiva' como criterio para la selección de voces:

El jesuita optó por una solución 'omnicomprensiva' ya que pensaba que el español, por su riqueza léxica y pasado prestigioso se podía proponer, en un momento de particular conflictividad con el francés (v. art. lengua), como lengua intermediaria de la cultura universal. La amplitud de su objetivo consistió en intentar «formar un Diccionario completo [con ciencias y artes], en cuanto me fuese posible, -afirma- no solo castellano, sino también de unos idiomas que le hiciesen universal en casi toda la tierra [...] se pone la voz de que se trata, con la definición más exacta que sea dable, y las voces Francesa, Latina e Italiana que correspondan al castellano» (VI) (San Vicente, 1995: 143).

En el medio siglo español -como he señalado al comienzo- concurren los proyectos que mejor definen el giro que se está operando en la diccionarística del español: por lo que hace a la lexicografía lingüística, el proyecto inconcluso de Juan Peñalver (Panléxico) ${ }^{21}$ y la exitosa edición del Diccionario nacional (1846$1847)^{22}$ de R. J. Domínguez; si atendemos a la lexicografía enciclopédica, la culminación del primer proyecto espańol: la Enciclopedia moderna (1851-1855). De mucho menor calado hemos de calificar las dos restantes obras destacadas por nuestro traductor: el Diccionario general de la lengua castellana (1849) bajo la dirección de José Caballero, pretendidamente enciclopédico pero que contó con una buena acogida editorial, y el Diccionario de la lengua castellana (1845)

19. Cosa bien distinta son las inexactitudes que el anónimo autor reprocha al padre Terreros. A saber, el peso de una libra por pieza y que el salto de esta especie sobre la superficie del mar tenga el alcance de un tiro de fusil, "pues lo que hace el volador es saltar unas tres varas del agua y correr hasta la distancia de un tiro de fusil» (Anónimo, 1852: col. 1009).

20. Diccionario nacional (1846-1847), Diccionario enciclopédico de la lengua española (18531855), Diccionario enciclopédico de la lengua castellana (1895) y Diccionario general y técnico hispano-americano (1918), respectivamente (cf. Real Academia Española, 2001).

21. El proyecto de Juan Peñalver constaba de once diccionarios diferentes, de los que tan solo llegaron a publicarse los cinco primeros: Diccionario universal de la lengua castellana (1842), Diccionario de sinónimos (1843), Vocabulario de fábula (1845), Diccionario etimológico (1845) y Diccionario de la rima (s. d.).

22. Es quizá el diccionario de nuestra lengua que más ediciones ha alcanzado, después del de la Academia: diecisiete en poco más de cuarenta años; la última, de 1889. Incluso se publicó un compendio, del que conozco cuatro ediciones aparecidas entre 1852 y 1887» (Seco, 2003: 291). 
de Prudencio J. de Vega, un diccionario manual con sendos suplementos sobre voces anticuadas y voces no reconocidas por la Real Academia.

La abundante producción de los años siguientes, no exenta de originalidad pero mimética en exceso, lleva incluso a la Real Academia Espańola a reorientar su concepción del diccionario oficial, algo que no había ocurrido en los últimos cien años. No fueron solo los acontecimientos editoriales y la pérdida de la hegemonía lexicográfica los factores decisivos para el cambio que se avecinaba, hemos de sumar la tímida aceptación por parte de la institución de un modelo de lengua diferente (lengua culta frente a lengua literaria) y la débil consciencia de un nuevo modo de enfrentarse a la descripción de los hechos de lengua (la lingüística histórico-comparada).

Incorpora nuestro traductor, en última instancia, una breve nota sobre la lexicografía bilingüe que se desarrolla por estos años, si bien solo referida a las lenguas española y francesa. Sin duda, como a nadie se le oculta, las que mayor interés despertaban en ese momento. La nota discurre por la mención de dos obras complementarias: un diccionario general y un diccionario fraseológico.

De una parte, el Nuevo diccionario francés-español y español-francés (1842), de autor anónimo ${ }^{23}$, un nuevo repertorio que se había redactado a partir del Dictionnaire français-espagnol et espagnol-français (1812) de Manuel Núñez de Taboada y con reproducción íntegra del prólogo de Antonio Capmany y Montpalau a su Nuevo diccionario francés-español (1805), las dos obras más representativas de nuestra lexicografía bilingüe en lo que llevábamos de siglo.

Y de otra, el Diccionario fraseológico español-francés y francés-español (1841), obra de Antonio Rotondo, quien nos ofrece, hasta donde alcanzan mis datos, una de las primeras obras, si no es que sea la primera, en su género, y cuyo modelo con toda probabilidad podrían haber sido los precedentes franceses para la lengua inglesa de J. Loffet (c. 1800) y G. Poppleton (1810 ${ }^{24}$.

A esta serie de referencias lexicográficas hemos de sumar las adiciones sobre el español de su tiempo, adiciones que vienen propiciadas por las notaciones del original francés: (1) la incorporación al fondo general del español, al igual que ha ocurrido en francés y en inglés, de la nueva terminología generada por los más recientes avances científico-técnicos; (2) el rechazo al galicismo innecesario «siendo como es el idioma de Cervantes el más rico y abundante de cuantos se hablan en Europa» (Anónimo, 1852: col. 1018) y (3) el apoyo gubernamental, si la Real Academia dejara de cumplir sus funciones, a personas y proyectos consagrados a la defensa de la lengua nacional: «al gobierno incumbe proteger los esfuerzos individuales, si ve que las personas que se deciden a acometer una empresa de esta magnitud, ofrecen todas las garantías de saber y de inteligencia que para ella se requiere» (Anónimo, 1852a: col. 1019).

23. [s. a.] (1842): Nuevo diccionario francés-español y español francés, 2. ${ }^{\mathrm{a}}$ ed., Barcelona, 2 tomos.

24. Loffet, J. (c. 1800): Phraséologie française et anglaise, Paris y Poppleton, Georg H. (1810): Phraséologie française et anglaise, ou dictionnaire de locutions françaises et anglaises, dont l'acception est la même, et la manière de les exprimer différente, Paris, Briand, respectivamente (cf. Quemada, 1967: 596 y 600). 


\section{Conclusiones}

El grado de equivalencia, pues, entre ambos textos viene determinado por el principio de invariación aplicado por el traductor, no en vano nos encontramos ante un texto científico-técnico (variante humanística) donde el método traductor suele discurrir mucho más próximo a la traducción verbum pro verbo que a la traducción sensum ad sensu.

El proceso de adecuación podríamos calificarlo de moderado, puesto que la producción lexicográfica de entonces era mucho más amplia que la reseñada por el anónimo autor, quien demuestra, como hemos visto, un buen conocimiento tanto de la lexicografía etimológica como de la lexicografía bilingüe entre las lenguas española y francesa; si bien es cierto, las notas referidas a los diccionarios españoles por géneros viene predetermiada, en buena medida, por el artículo original francés.

La Enciclopedia moderna, como ya he señalado, fue el primer repertorio de factura española que logró culminarse con éxito. De la recepción de esta obra dieron cumplida cuenta, bien que notablemente resumidos, los artículos diccionario de dos de las enciclopedias más representativas de la segunda mitad del siglo XIX español: en el tomo V (Anónimo 1876) del Diccionario universal de la lengua castellana (1875-82), dirigido por Nicolás M. a Serrano, y en el tomo VI (Anónimo 1890) de la obra publicada por los editores catalanes Montaner y Simón, Diccionario enciclopédico hispanoamericano de literatura, ciencias y artes (1887-1898).

No tuvo mejor suerte el trabajo de J. B. Pellissier: la segunda parte de su artículo («Principales conditions d'un bon dictionnaire») quedó reducida a solo la introducción en el tomo 7 (1860) de la segunda edición del Dictionnaire de la conversation et de la lecture (1853-1860), con la consiguiente devaluación de la meritoria tarea de su autor ${ }^{25}$. Todo el apartado teórico y la sistematización de los problemas que plantea un diccionario de lengua se condenan al silencio cuando se trata en realidad de la parte más provechosa del trabajo de J. B. Pellissier. En teoría lexicográfica habrá que esperar al desarrollo de la semántica en los años sesenta del pasado siglo para que se retomen teóricamente los grandes problemas que aquejan a la confección de diccionarios, ya sean monolingües, como nos plantea J. B. Pellissier, ya bilingües o plurilingües.

Hemos de reconocer, en definitiva, que la traducción y adecuación al español del trabajo de J. B. Pellissier debe figurar -hasta donde alcanzan mis datos- como el primer testimonio de cierta entidad teórica en nuestro pasado metalexicográfico. No debemos olvidar, de igual forma, que la renovación de parte de la lexicografía española extraacadémica de entonces -como aquí se ha

25. Bajo la firma de L. Lovet se incorpora, con el fin de compensar tan decisiva supresión para el conocimiento de la metalexicografía monolingüe, una actualización sobre los diccionarios franceses publicados en las últimas décadas. 
señalado- procedía de algunos de los proyectos de mayor calado desarrollados en Francia: el Pan-lexique de Ch. Nodier y el Dictionnaire national de L. N. Bescherelle por lo que respecta a la lexicografía lingüística; el Dictionnaire de la conversation et de la lecture y la Encyclopédie moderne (de L. Renier) si atendemos a la lexicografía enciclopédica.

\section{Referencias}

Ahumada Ignacio, "Bibliografía de la metalexicografía del español: la crítica de diccionarios (ss. XVI al XIX), en Campos Soto Mar, Cotelo García Rosa \& Pérez Pascual José Ignacio (ed.), Historia de la lexicografía española, A Coruña, Universidade da Coruña, 2007, p. 9-19.

Ahumada Ignacio, «De los primeros tratados lexicográficos del español (siglos XVIIIXIX)», en Azorín Fernández Dolores y otros (ed.), El Diccionario como puente entre las lenguas y culturas del mundo, Alicante, Universidad de Alicante, 2008, p. 41-45.

Ahumada Ignacio, «El DRAE como género metalexicográfico: la duodécima edición o de 1884», en Vázquez Laslop M. ${ }^{a}$ Eugenia, Zimmermann Klaus \& Segovia Francisco (ed.), De la lengua por solo la extrañeza. Estudios de lexicología, norma lingüistica, historia y literatura en homenaje a Luis Fernando Lara, México, El Colegio de México, 2011, I, p. 57-74.

Ahumada Ignacio, «Enciclopedia y diccionario de lengua: el estatuto lexicográfico del Tesoro", Académica, 6, 2011a, p. 19-29.

Anónimo, "Diccionario», en Enciclopedia Moderna, Madrid, Establecimiento Tipográfico de Mellado, 1852, XIII, cols, 997-1020.

Anónimo, «Diccionario», en Serrano Nicolás M.a (dir.), Diccionario universal, Madrid, Astort hermanos, editores, 1876, v, p. 422-23.

Anónimo, «Diccionario», en Diccionario enciclopédico hispano-americano de literatura, ciencias y artes, Barcelona, Montaner y Simón, editores, 1890, IV, p. 565-568.

Grases Pedro, «Del porqué no se escribió el Diccionario matriz de la lengua castellana de Rafael María Baralt», Caracas, Escuela Técnica Industrial-Talleres de Artes Gráficas, 1943.

Hausmann Franz Josef, «Pour une histoire de la métalexicographie», en Hausmann Franz Josef, Reichmann Otto, Weigand Hert Ernst \& Zgusta Ladislav (ed.), Wörterbücher. Dictionaries. Dictionnaires. Ein internationales Handbuch zur Lexicographie. An International Encyclopaedia of Lexicography. Encyclopédie internationale de lexicographie, Berlin-New York, De Gruyter, 1989, I, p. 216-224.

Martínez Egido José Joaquín, «El Dizionario italiano-spagnuolo e spagnuolo-italiano (1844-1847) de Martínez del Romero», en San Vicente Félix (dir.), Textos fundamentales de la lexicografia italo-española (1805-1916), Monza, Polimetrica International Scientific Publisher, 2010, p. 115-146.

Mellado Francisco de Paula, «Prólogo», en Enciclopedia moderna, Madrid, Establecimiento Tipográfico de Mellado, 1851, p. V-IX.

Pellissier Jean Baptiste, «Dictionnaire», en Dictionnaire de la conversation et de la lecture, Paris, Belin-Mandar, 1835, XX, p. 468b-485a. 
Pellissier Jean Baptiste, «Recherches sur les anciens lexiques, suivies de considérations sur les principaux moyens d'améliorer les nouveaux dictionnaires. Première partie: Des anciens lexiques et des principaux vocabulaires modernes», en Bulletin du Bibliophile, 1836, 4 (2.a série), p. 119-138.

Pellissier Jean Baptiste, «Recherches sur les anciens lexiques, suivies de considérations sur les principaux moyens d'améliorer les nouveaux dictionnaires. Deuxième partie: De la composition d'un dictionnaire», en Bulletin du Bibliophile, 1836a, 5 (2. ${ }^{a}$ série), p. 167-181.

Quemada Bernard, Les dictionnaires du français moderne. 1539-1863. Etude sur leur histoire, leurs types et leurs méthodes, Paris, Didier, 1967.

Quérard Joseph Marie, La France littéraire, ou dictionnaire bibliographique des savants, historiens et gens de lettres de la France, Paris, Chez Firmin Didot frères, 1835, VII.

Real Academia Española, Nuevo tesoro lexicográfico de la lengua española (edición en dvd-rom), Madrid, Espasa-Calpe, 2001.

San Vicente Félix, "Innovación y tradición en el Diccionario (1786-1793) de E. de Terreros y Pando», en Pantaleoni Luisa, Salmon Kovarski Laura (ed.), Sapere linguistico e sapere enciclopedico, Atti del Convegno Internazionale svoltosi a Forli dal 18 al 20 Aprile 1994, Bolonia, CLUEB, 1995, p. 139-158.

Seco Manuel, Estudios de lexicografía española, Madrid, Editorial Gredos, 2003.

Zamora Vicente Alonso, La Real Academia Española, Madrid, Espasa, 1999. 